IdeAs

Idées d'Amériques

14 | 2019

Populismes dans les Amériques

\title{
Pourquoi il est urgent de relire Whitman
}

\section{Kenneth M. Price}

Traducteur : Anne Reynès Delobel

\section{CpenEdition}

\section{Journals}

Édition électronique

URL : http://journals.openedition.org/ideas/6750

DOI : $10.4000 /$ ideas. 6750

ISSN : 1950-5701

Éditeur

Institut des Amériques

Référence électronique

Kenneth M. Price, « Pourquoi il est urgent de relire Whitman », IdeAs [En ligne], 14 | 2019, mis en ligne le 01 octobre 2019, consulté le 08 novembre 2019. URL : http://journals.openedition.org/ideas/6750 ; DOI : 10.4000/ideas.6750

Ce document a été généré automatiquement le 8 novembre 2019.

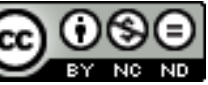

IdeAs - Idées d'Amériques est mis à disposition selon les termes de la licence Creative Commons Attribution - Pas d'Utilisation Commerciale - Pas de Modification 4.0 International. 


\title{
Pourquoi il est urgent de relire Whitman
}

\author{
Kenneth M. Price \\ Traduction : Anne Reynès Delobel
}

1 Que penser de Whitman et de son héritage à l'heure où nous célébrons le bicentenaire de sa naissance ? À cette occasion, pléthore d'expositions, de conférences, de lectures et de célébrations ont été organisées aux États-Unis et de par le monde. Whitman est en effet l'un des rares écrivains du XIX ${ }^{e}$ siècle qui continuent d'exercer une influence sur l'opinion publique. Comment expliquer cette popularité jamais démentie (en particulier aux États-Unis, mais également à l'étranger), notamment dans les campagnes publicitaires, les émissions de télévision, le cinéma et les discours politiques? Pourquoi Whitman suscite-t-il encore aujourd'hui autant d'intérêt? La réponse se trouve bien entendu dans son œuvre mais, plus encore, dans le sens qu'on lui a donné à travers sa réception et ses transformations, par-delà les frontières, au fil des décennies et désormais des siècles. Si Whitman nous parle aujourd'hui, c'est aussi parce que de «nouveaux » écrits de sa main ne cessent de refaire surface. Au cours des dernières années, un volume impressionnant d'archives et de documents inédits a été mis au jour : un roman dont on ignorait jusqu'alors l'existence (Jack Engle), un manuel de santé à l'usage du citadin, publié sous un pseudonyme (Manly Health and Training), des poèmes de jeunesse jusqu'ici non-identifiés, ainsi que d'autres écrits (lettres, carnets, essais sur l'art, etc.). Loin d'être l'apanage des chercheurs, ces documents ont été mis en ligne et sont accessibles au grand public ${ }^{1}$.

2 Les commémorations qui ont marqué ce bicentenaire n'ont pas toutes donné lieu à un concert d'éloges. Cette année, la revue North American Review a fait paraitre une brillante série, intitulée « Every Atom » sous la forme de deux cents brèves annotations du chef-d'œuvre de Whitman, "Chanson pour moi-même», rédigées par divers auteurs, parmi lesquels des poètes, des activistes, des physiciens, des astronomes, des artistes et des défenseurs de l'environnement. Si la plupart de ces commentaires portent Whitman aux nues, d'autres sont beaucoup plus critiques. On a longtemps voulu voir en Whitman un défenseur de la liberté artistique, politique et sexuelle, et 
lorsqu'il s'avère que ses écrits ne sont pas en accord avec ces principes, d'aucuns ont tendance à se croire abandonnés, voire trahis. Whitman a souvent été rangé parmi les pères du multiculturalisme américain. Sa poésie, qui recourt volontiers à l'argot et mélange les langues, se projette en imagination vers d'autres pays et rejette toute forme de discrimination, apporte un solide argument en faveur de cette thèse. Toutefois, elle fait également entendre la voix d'une culture fondée sur l'esclavage, le génocide et les politiques discriminatoires. Dans le climat actuel, marqué par l'intolérance ethnique, raciale et religieuse, il n'est guère étonnant que la pensée et l'héritage de l'écrivain fassent l'objet d'un surcroît d'attention.

3 Le débat achoppe sur une question dont la réponse se trouve peut-être dans la volumineuse œuvre en prose: comment se fait-il que Whitman, qui a su si éloquemment prêter voix à la diversité américaine pour célébrer les multitudes foisonnantes et variées qui composent "une nation de nations ", n'ait pas soutenu la cause des Afro-Américains à des moments-clés de leur histoire ? N'avait-il pas en effet affirmé qu'il ferait entendre la voix de celles et ceux qui n'avaient jamais eu droit à la parole?

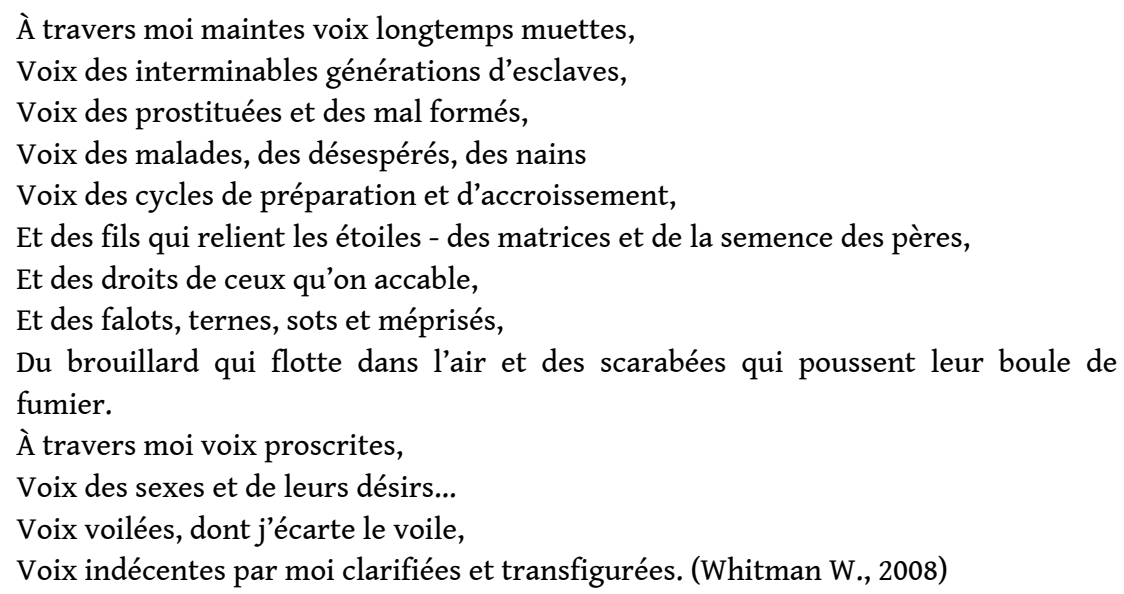

Pendant la guerre de Sécession, Whitman rassemble des coupures de journaux relatant la contribution déterminante des Afro-Américains à l'effort de guerre de l'Union, mais sa poésie n'en porte pas trace. L'écrivain ne s'exprime pas non plus sur la question de l'émancipation, au contraire de Charlotte Forten qui parle du « jour le plus glorieux de notre nation » (Forten C., 1988: 428). Il garde également le silence sur le Quinzième Amendement de la Constitution des États-Unis qui dit, quoique de manière négative, que le droit de vote des citoyens des États-Unis «ne sera refusé pour des raisons de race, couleur ou de condition antérieure de servitude ». En outre, il emploie rarement le terme « Reconstruction » qui connote un changement social radical. Cette réticence de Whitman à s'engager en faveur du droit de vote des Noirs demeure l'un des aspects dérangeants de sa carrière. Elle est d'autant plus surprenante que l'amitié entre les races était l'une des sources d'inspiration majeures de ses premiers poèmes. À plusieurs reprises Whitman forme le vœu que les problèmes qu'il associe aux Afro-Américains se résoudront d'eux-mêmes.

5 Au cours de la guerre, Whitman soigne surtout les soldats blancs du Nord et du Sud, bien qu'il lui arrive d'apporter aide et réconfort aux soldats afro-américains. Dans Memoranda During the War (1875), il rapporte : « dès que cela était possible, je me suis aussi porté au chevet des soldats noirs, blessés ou malades, ou dans les campements de contrebande, faisant de mon mieux pour les soulager». Le terme «contrebande de 
guerre » est utilisé dès le début du conflit pour désigner les esclaves fugitifs qui choisissent de s'engager dans les rangs de l'Union et que le général Benjamin Butler refusera de rendre aux sudistes lorsqu'ils réclameront qu'on leur rende leurs " possessions ». Dès lors, on aura coutume de parler des soldats noirs de l'Union comme de la « contrebande ». Quant aux campements de contrebande, ils sont réputés pour la pauvreté et les conditions sanitaires déplorables qui $\mathrm{y}$ règnent. Les épidémies qui $\mathrm{y}$ sévissent rendent leur fréquentation dangereuse. Aussi, le bénévolat de Whitman auprès des Noirs, dans les campements de contrebande ou les hôpitaux, est apprécié à sa juste mesure. À l'occasion du soixante-dixième anniversaire de Whitman, Horace Traubel relate, dans With Walt Whitman in Camden, qu'une "domestique noire " se précipite pour saluer chaleureusement le poète. Au souvenir de cette rencontre, Whitman se remémorera aussi ses visites fréquentes «à un hôpital pour nègres [...] à Culpepper » (Traubel H. $1964:$ 299).

Près de dix ans après la fin du conflit, Whitman note dans " 'Tis But Ten Years Since »:

Pour moi, la guerre, abdiquant toute grandiloquence historique, et entièrement épargnée par la question de l'Esclavage, se rapporte à ces pages miniatures [ses carnets d'hôpitaux] [...] ce ne sont pas des mots, mais des formules magiques [...] Elles convoquent, dans la pièce vide et silencieuse où j'écris [...] les fantômes innombrables de ceux qui sont tombés et ont été hâtivement ensevelis en masse sur les champs de bataille, ou ceux dont la poussière et les ossements ont été depuis transférés dans les cimetières nationaux, dans toute la Virginie et le Tennessee. (Whitman W., $1874: 3$ )

7 L'engagement bénévole de Whitman auprès de milliers de soldats blessés, isolés et désorientés fut en tout point remarquable. Pour lui témoigner leur gratitude, nombre d'entre eux choisiront de donner son prénom à leurs enfants. D'autres ont dit qu'ils lui devaient la vie. L'attention qu'il portait à ces hommes donnait, de fait, des résultats extraordinaires. Le docteur Willard Bliss, chirurgien en chef de l'hôpital des armées (Armory Square) à Washington, qui enregistrait le taux de mortalité le plus élevé et où Whitman passait le plus clair de son temps, a déclaré : «d'après ce que j'ai pu personnellement observer du travail que M. Whitman a accompli à Armory Square et dans d'autres hôpitaux, je suis d'avis qu'aucun autre bénévole n'a accompli autant auprès de soldats et du gouvernement que $M$. Whitman ». Si l'on ne peut mettre en doute la portée humanitaire de l'action bénévole de Whitman, il convient cependant de déceler la part de fantasme qui entre dans son désir de faire de ses carnets des «formules magiques" capables de résumer les bouleversements politiques et sociaux de l'époque. Le verbe "abdiquer» est de ce point de vue révélateur car il indique tout autant la conscience que le poète a de sa responsabilité (lui qui ambitionne de devenir le barde de la nation) face aux immenses changements en train de se produire (près de trois millions d'esclaves viennent d'être émancipés) que de son choix délibéré d'échapper à ses devoirs.

8 Il est curieux d'observer qu'au cours des années qui suivent le conflit, l'intérêt que Whitman porte aux Afro-Américains ne cesse de diminuer. De 1865 à 1873, il occupe un emploi de bureau dans le cabinet du Procureur Général à Washington. Pendant ses heures creuses ou de loisir, il se consacre à sa correspondance, gère ses affaires littéraires et médite sur l'avenir de la démocratie. À cette même période, il s'attache à l'écriture de Perspectives démocratiques (1871), où il entend livrer ses réflexions sur l'avenir des États-Unis dans la veine des grands critiques britanniques, tels Matthew Arnold ou Thomas Carlyle. Ces «perspectives» démocratiques, il peut les contempler 
depuis la fenêtre de son bureau : à l'ouest, il voit l'avenir du pays se dérouler «vers l'intérieur du pays et en direction de l'océan pacifique » (Whitman W., $1860: 371$ ). Au sud, au-delà du Potomac, il aperçoit les collines de la Virginie, gorgées de sang, ce qui l'amène à réfléchir aux ravages causés par le conflit qui vient de s'achever et à ses lendemains troublés, notamment marqués par l'émergence du Ku Klux Klan, l'un des sujets récurrents de sa correspondance. De fait, au vu de la place qu'il accorde à la question du Klan, on peut s'étonner que Whitman ne réagisse pas de manière plus énergique et plus directe à la diatribe raciste de Carlyle dans "Shooting Niagara ", un texte auquel Perspectives démocratiques tente de répondre sans pour autant y parvenir. Carlyle avait déploré que "un demi-million d'excellents Blancs [...] dotés des plus grands talents et des facultés les plus remarquables, se soient entretués de la manière la plus atroce [...] tandis que trois millions de Noirs ineptes [...] se voient complétement « émancipés » et lancés sur la voie du progrès » (Carlyle T., 1867). L'historien écossais considérait en effet que le droit de vote de 1867 («Reform Act») représentait un danger pour l'Angleterre et que les États-Unis commettraient un suicide culturel s'ils accordaient le droit de vote aux Noirs (le titre de son pamphlet fait référence aux cassecou qui dévalaient les chutes du Niagara dans un tonneau).

Or, si Whitman promet dans la préface de Perspectives démocratiques de répondre à Carlyle, force est de constater qu'il ne parvient pas vraiment à ses fins et préfère éviter la question-encore un silence de sa part. Ce n'est que dans les notes placées à la fin de l'ouvrage qu'il déclare : «Quant au suffrage universel, après tout, puisque nous sommes allés si loin, plus il sera généralisé, mieux ce sera. Je suis en faveur de l'ouverture des portes la plus large qui soit. Que le vannage se fasse sur l'aire la plus large possible et tout ira bien » (Whitman W., 2016:224). Cette affirmation ne laisse pas d'être étrange. Whitman propose que le droit de vote soit étendu non seulement aux anciens esclaves noirs, mais aussi aux femmes et aux Amérindiens. Cependant, la rhétorique de la fraternité que l'on retrouve dans les poèmes consacrés à la démocratie cède ici le pas à un langage plus scientifique emprunté aux discours hygiénistes de l'époque selon lesquels les maladies étaient véhiculées par la pauvreté et la surpopulation qui régnaient dans les hôpitaux, les taudis urbains et les camps de contrebande. Whitman considère que chacun pourra voter sans risque pour sa santé lorsque le pays aura résolu ces problèmes d'hygiène publique. On est bien loin d'« Au-dessus du carnage une voix s'éleva » où le poète affirme, quoique de manière vague, que «l'affection encore viendra résoudre les problèmes de la liberté » (Whitman W., 1865 :49).

Pour le dire vite, Whitman peut encore parler le langage de l'universalisme poétique et démocratique, et de l'adhésion aux idéaux démocratiques, tout en évitant de traiter la question raciale. Et lorsqu'il mentionne spécifiquement cette question, c'est pour l'esquiver. Voilà pourquoi aujourd'hui de nombreux critiques se déclarent déçus par Whitman. Le poète et critique Lavelle Porter a récemment demandé, en manière de provocation : "Faut-il \#zapper Walt Whitman? ». La question de Porter nous pose un défi, particulièrement à un moment où d'aucuns s'inquiètent de l'écho favorable que le suprématisme blanc semble trouver auprès d'une partie non négligeable de la population du pays. Porter fait référence à un incident, survenu en 2013 à l'Université Northwestern, lorsqu'un étudiant noir et gay inscrit en master de musicologie avait refusé d'interpréter une composition inspirée de la poésie de Whitman au motif que l'écrivain était raciste. Porter écrit :

J'espère que nous pouvons encore rendre hommage à Whitman sans pour autant nous voiler la face sur ses défauts ni sur ceux de l'Amérique. Comme le dit June 
Jordan : «Moi aussi, je suis une descendante de Walt Whitman. Moi aussi, je cherche à dire la vérité au sujet de l'histoire de ces terres qui ont vu couler tant de sang, de toutes ces choses que nous devrions considérer comme sacrées et de toutes celles que certains se vantent d'avoir profanées et anéanties ». ${ }^{2}$

11 Langston Hughes, qui avait décidé, dans un geste demeuré célèbre, de ne pas jeter Feuilles d'herbe par-dessus le bastingage du paquebot qui le conduisait en Afrique (il a choisi de se délester d'autres aspects de la culture occidentale) nous invite à une approche plus magnanime lorsqu'il nous incite à garder le meilleur chez autrui, et en particulier chez Whitman. Alors que nous traversons à nouveau une période marquée par de vives tensions raciales, juger trop sévèrement Whitman, c'est aussi courir le risque d'oublier qu'il fut aussi et avant tout un poète qui a célébré l'espoir et un monde d'infinies possibilités, et qui a su inspirer nombre de progressistes aux États-Unis et à travers le monde.

Dans le cas de Whitman, le lien entre l'homme et l'artiste est une question capitale. L'écrivain avait compris qu'en créant le «Walt Whitman » des Feuilles d'herbe, il avait inventé une version idéalisée de lui-même, distincte de celle de l'individu Walt Whitman. Pour certains critiques, cette distance est un signe de mauvaise foi, d'hypocrisie, de duplicité ou de manque de sincérité. Mais faut-il regretter que dans ses plus beaux écrits Whitman ait cherché à exprimer un point de vue plus généreux, plus bienveillant et plus tolérant que ceux qu'il pouvait lire et se laissait parfois aller à exprimer dans ses écrits journalistiques (de moins bonne facture), dans des notes hâtivement griffonnées dans ses carnets, dans ses lettres à ses proches ou à des soldats issus de la classe ouvrière et (quoique plus rarement) dans les points aveugles de sa production poétique? À sa décharge, Whitman s'est, la plupart du temps, efforcé de s'élever au-dessus des préjugés de son époque.

Si Whitman présente tant de difficultés à l'interprétation, c'est que chez lui, le personnage fictif «Walt Whitman » et le vrai Walt Whitman semblent souvent ne faire qu'un. Toutefois, certains aspects du personnage (il se targue ainsi de sa grossièreté et de son manque de manières, ainsi que d'avoir engendré une nombreuse descendance) ne s'accordent pas avec les faits biographiques. Le «moi» projeté de Whitman est toujours complexe, ample, contradictoire et, en fin de compte, jamais univoque. Brian Clements a écrit :

Avec Whitman, c'est une lutte continuelle et il ne cesse de nous échapper, de même que la question de l'identité (individuelle ou nationale) nous échappe. À l'instar de Whitman, [les États-Unis] sont une énorme contradiction: une nation investie d'espoir, mais qui s'avère incapable d'offrir les services les plus basiques, comme les soins médicaux ou l'accès à l'eau potable, à l'ensemble de ses citoyens ; une nation qui prétend être fondée en droit, mais où l'injustice règne quotidiennement en maître. Si « les États-Unis sont le plus grand poème » comme l'affirme Whitman au début de sa préface de l'édition de 1855 des Feuilles d'herbe, alors ce grand poème est à la fois un beau gâchis et le souvenir falot de la possibilité de renouveau, de justice et de réconciliation. ${ }^{3}$

14 La perspective politique et culturelle que nous donne Whitman est tour à tour inspirée et myope, et parfois entachée par son penchant pour les politiques nativistes et les théories raciales pseudo-scientifiques. Pourtant, alors même que nous déplorons ces errances de Whitman, nous lui reconnaissons un rôle de rebelle, d'autodidacte, de marginal, et saluons le poète qui a dit avec insistance que l'esclave est l'égal du maître. L'héritage de Whitman est brouillon et complexe, à l'image de ce pays qui peut tout à la 
fois susciter chez nous le découragement le plus profond et l'enthousiasme le plus ardent.

Actuellement, aux États-Unis, le pourcentage de détenus issus de la population noire est supérieur à celui enregistré en Afrique du Sud au plus fort de l'apartheid. Dans la capitale du pays, Washington D.C., près de trois jeunes Afro-Américains sur quatre (issus pour la plupart des quartiers les plus défavorisés) passeront un jour par la case prison. La majorité d'entre nous ne se sent pas concernée par ce triste constat. Voilà pourquoi, avant de reprocher à Whitman ses silences et son absence de mobilisation, nous devrions peut-être nous livrer à un examen de conscience.

\section{BIBLIOGRAPHIE}

Carlyle, Thomas, “Shooting Niagara-And After?", Macmillan's Magazine, Edinburgh, Vol. XVI, April 1867.

Forten, Charlotte L, The Journals of Charlotte Forten Grimké, Brenda Stevenson (ed.), New York and Oxford, Oxford University Press, 1988.

Traubel, Horace, With Walt Whitman in Camden vol. 5 (April 8 - September 14, 1889), Gertrude Traubel (ed.), Carbondale, Illinois, Southern Illinois University Press, 1964, p. 299. Also available online: https://whitmanarchive.org/criticism/disciples/traubel/WWWiC/5/whole.html

Whitman, Walt, Feuilles d'herbe, traduit de l'anglais (États-Unis) par Éric Athenot, [ed. orig. Leaves of Grass, Brooklyn, New York, 1855], Paris, José Corti, 2008.

Whitman, Walt, "Calamus" in Leaves of Grass, 1860 edition.

Whitman, Walt, Drum-Taps, New York, 1865.

Whitman, Walt, “'Tis Ten Years Since”, New York Weekly Graphic, 24 January 1874, p. 3.

Whitman, Walt, Manuel d'Amérique, traduit de l'anglais (États-Unis) et préfacé par Éric Athenot, Paris, Corti, 2016.

Whitman, Walt, Memoranda During the War (Camden, NJ, 1875-1876).

https://whitmanarchive.org/published/other/memoranda.html

\section{NOTES}

1. Note de la rédaction : le site Walt Whitman Archive, co-dirigé par Ed Folson et Kenneth Price, publié par le Center of Digital Reseach Humanities (University of Nebraska-Lincoln), est accessible à partir du lien suivant : https://whitmanarchive.org/.

2. Lavelle Porter, "Should Walt Whitman Be \#Cancelled?" JSTOR Daily, April 17, 2019.

3. Brian Clements, "Introduction," in Every Atom: reflections on Walt Whitman at 200, North American Review, May 30, 2019. 


\section{AUTEURS}

\section{KENNETH M. PRICE}

Kenneth M. Price is Co-Director of the Walt Whitman Archive and Hillegass University Professor of American Literature at the University of Nebraska-Lincoln. kprice2@unl.edu 